\title{
Botanical species giving unifloral honey in Europe
}

\author{
Livia Persano Oddo a*, Lucia Piana ${ }^{\mathrm{a}}$, Stefan Bogdanov ${ }^{\mathrm{b}}$, Antonio Bentabol ${ }^{\mathrm{c}}$, \\ Panagiota GOTSIOU $^{\text {d }}$, Jacob KerKVLIET ${ }^{\mathrm{e}}$, Peter MARTIN ${ }^{\mathrm{f}}$, Monique MORLOT' \\ Alberto ORTIZ VALBUENA ${ }^{\mathrm{h}}$, Kaspar RUOFF ${ }^{\mathrm{b}}$, Katharine VON DER OHE ${ }^{\mathrm{i}}$ \\ ${ }^{\text {a }}$ Istituto Sperimentale per la Zoologia Agraria, Sezione di Apicoltura, Roma, Italy \\ ${ }^{b}$ Federal Dairy Research Station, Swiss Bee Research Centre, Liebefeld, Switzerland \\ ${ }^{\mathrm{c}}$ Casa de la miel, Tenerife, Canarias, Spain \\ ${ }^{d}$ Laboratory of Analytical Chemistry, Mediterranean Agronomic Institute of Chania, Crete, Greece \\ e Inspectorate for Health Protection and Veterinary Public Health, Amsterdam, The Netherlands \\ ${ }^{\mathrm{f}}$ Honey International Packers Association, London, UK \\ g Bernard Michaud S.A., Jurancon, France \\ h Centro Regional Apicola, Guadalajara, Spain \\ i Niedersächsisches Landesinstitut für Bienenkunde, Celle, Germany
}

(Received 20 February 2004; revised 11 May 2004; accepted 17 May 2004)

unifloral honey / Europe / botanical species

In Europe more than one hundred botanical species can give unifloral honeys. Most of them have only a local importance and are thus marketed on a limited scale, but have also been described in the literature.

To complete the information relating to European unifloral honeys (Persano Oddo and Piro, 2004), the International Honey Commission (IHC) carried out an extensive survey of all these botanical species, partly collecting data from bibliographic references and partly using information directly provided by the IHC members (the authors of this study).

All the botanical species giving unifloral honeys in Europe are listed in Table I, where the scientific name is reported together with local names in 6 European languages. Information is given on their respective importance for honey production in the various European countries, following a subdivision into 3 classes: abundant, medium and rare. In some cases the information relating to a country is extended to a wider surrounding geographical area. Where possible, references are given for each botanical species, where the reader can find data on the production and properties of the respective honey types.

\footnotetext{
* Corresponding author: livia.persano@ apicoltura.org
}

Honeys from different species belonging to the same botanical genus have sometimes similar characteristics, and they were considered together in the descriptive sheets (Persano Oddo and Piro, 2004). Here, as far as possible, all individual species are considered.

The honeydew honeys need special attention. Chemically they form two main groups: honey from honeydew produced by Metalfa pruinosa (Say) and other honeydew honeys (Persano and Piro, 2004). Actually, honeydew honeys are produced by many different aphids and insects on a big variety of plants, mostly trees (coniferous and latifoliae). The main coniferous honeydew honeys are produced from fir, spruce and pine trees. They are highly valued in different European countries and are marketed with specific denominations. Latifoliae honeydew honeys form a rather large group. Most of them, with the exception of French oak honey (Gonnet and Vache, 1998) are not characterised and are marketed in central Europe as "forest" honey, often mixed with honeydew honeys from coniferous trees. There are good overview references on their production (Kloft and Kunkel, 1985; Liebig, 1999) and properties (Maurizio, 1965; Crane and Walker, 1985; Maurizio, 1985). 
Table I. List of botanical species giving unifloral honeys in Europe.

Legend: A = Austria; $\mathrm{AL}$ = Albania; B = Belgium; BG = Bulgaria; BI = British Islands; $\mathrm{CH}=$ Switzerland; D = Germany; DK = Denmark; E = Spain; East-Eu = Includes: Czech Rep, Slovak

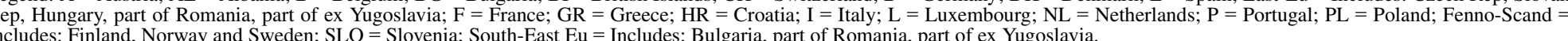
$=$ Produced in the Canary islands.

\begin{tabular}{|c|c|c|c|c|c|c|c|c|c|c|}
\hline Latin name & English & French & Italian & German & Spanish & Finnish & Abundant & Medium & Rare & References \\
\hline $\begin{array}{l}\text { Ailanthus altissima } \\
\text { (Miller) Swingle }\end{array}$ & $\begin{array}{l}\text { Tree of } \\
\text { heaven }\end{array}$ & Ailante & Ailanto & Götterbaum & Ailanto & Jumaltenpuu & & & I, E, D, F & $\begin{array}{l}\text { Maurizio and Schaper, 1994; } \\
\text { Persano Oddo et al., } 2000\end{array}$ \\
\hline Allium сера $\mathrm{L}$. & Onion & Oignon & Cipolla & $\begin{array}{l}\text { Gemeine } \\
\text { Zwiebel }\end{array}$ & Cebolla & Sipuli & & & $\begin{array}{c}\text { E, D, NL, CH, } \\
\text { I, F }\end{array}$ & $\begin{array}{l}\text { Maurizio and Louveaux, 1965; } \\
\text { Maurizio and Schaper, 1994; } \\
\text { Persano Oddo et al., } 2000\end{array}$ \\
\hline Amorpha fruticosa $\mathrm{L}$. & Bastard indigo & & $\begin{array}{l}\text { Indaco } \\
\text { bastardo }\end{array}$ & Bastardindigo & Falso índigo & Sulkapensas & & & $\begin{array}{l}\text { I, South-East } \\
\text { Eu }\end{array}$ & $\begin{array}{l}\text { Ricciardelli D'Albore, 1998; } \\
\text { Persano Oddo et al., } 2000\end{array}$ \\
\hline Anthyllis cytisoides $\mathrm{L}$. & & $\begin{array}{l}\text { Anthyllide } \\
\text { faux-cyprès }\end{array}$ & & Wundklee & Albaida & & & E & & $\begin{array}{l}\text { Gonnet and Vache, 1998; Sáenz Laín } \\
\text { and Gómez Ferreras, } 2000\end{array}$ \\
\hline Arbutus unedo $\mathrm{L}$. & Strawberry tree & Arbousier & Corbezzolo & Erdbeerbaum & Madroño & $\begin{array}{l}\text { Lännenmansi } \\
\text { kkapuu }\end{array}$ & & $\mathrm{I}, \mathrm{F}, \mathrm{AL}, \mathrm{HR}$ & $\mathrm{E}, \mathrm{GR}$ & $\begin{array}{l}\text { Ricciardelli D’Albore, 1985, 1998, } \\
\text { 2003; Prota et al., 1997; Persano } \\
\text { Oddo et al., 2000; Sáenz Laín and } \\
\text { Gómez Ferreras, 2000; Clément, } \\
2002\end{array}$ \\
\hline Asparagus spp. & Asparagus & Asperge & Asparago & Spargel & Espárrago & Parsa & & & $\begin{array}{l}\text { I, DK, NL, D, } \\
\text { F, CH, HR }\end{array}$ & $\begin{array}{l}\text { Maurizio and Schaper, 1994; } \\
\text { Persano Oddo et al., 2000; } \\
\text { Ricciardelli D'Albore, 1998; } 2003\end{array}$ \\
\hline $\begin{array}{l}\text { Asphodelus microcarpus } \\
\text { Salzm. et Viv. }\end{array}$ & Asphodel & Asphodèle & Asfodelo & Affodill & Asfodelo & & & I & & $\begin{array}{l}\text { Prota et al., 1997; Gonnet and Vache, } \\
\text { 1998; Ricciardelli D'Albore, 1998; } \\
\text { Persano Oddo et al., } 2000\end{array}$ \\
\hline Borago officinalis $\mathrm{L}$. & Borage & $\begin{array}{l}\text { Bourrache } \\
\text { officinale }\end{array}$ & Borragine & Borretsch & Borraja & Purasruoho & & & $\begin{array}{l}\text { I, E, NL, F, } \\
\text { Fenno-Scand, } \\
\text { BI }\end{array}$ & $\begin{array}{l}\text { Crane et al., 1984; Maurizio and } \\
\text { Schaper, 1994; Quayle, 1997; } \\
\text { Persano Oddo et al., } 2000\end{array}$ \\
\hline Brassica napus $\mathrm{L}$. & Rape & Colza & Colza & Raps & Colza & Rapsi & $\begin{array}{l}\text { CH, NL, East } \\
\text { Eu, D, B, DK, } \\
\text { Fenno-Scand, } \\
\text { L, BI, F }\end{array}$ & E & $\begin{array}{l}\text { I, South-East } \\
\text { Eu }\end{array}$ & $\begin{array}{l}\text { Maurizio and Louveaux, 1965; } \\
\text { Louveaux, 1970; Crane et al., 1984; } \\
\text { Maurizio and Schaper, 1994; Persano } \\
\text { Oddo et al., 2000; Sáenz Laín and } \\
\text { Gómez Ferreras, 2000; Clément, } \\
\text { 2002; Piazza and Persano Oddo, } 2004\end{array}$ \\
\hline
\end{tabular}




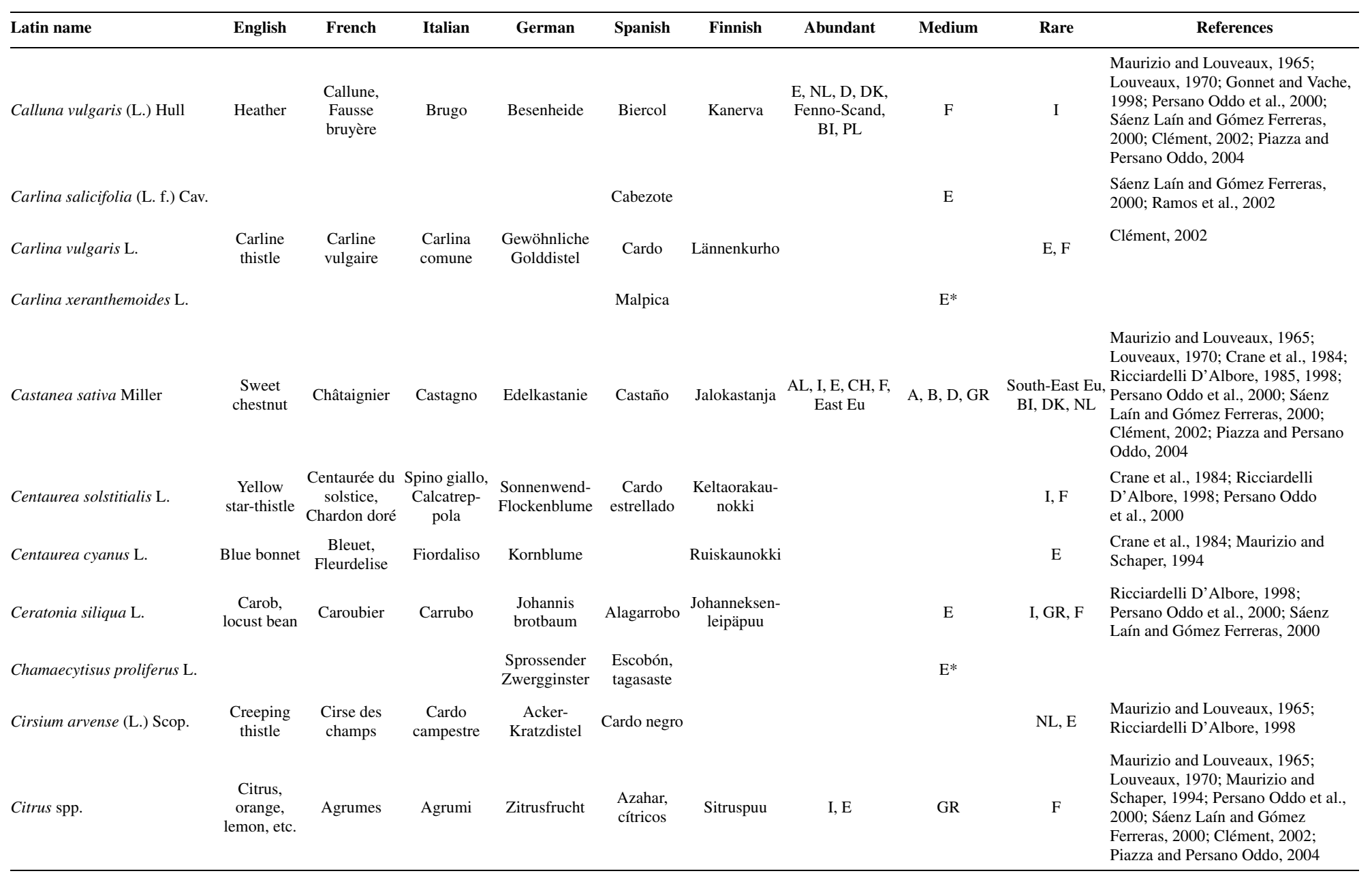




\begin{tabular}{|c|c|c|c|c|c|c|c|c|c|c|}
\hline Latin name & English & French & Italian & German & Spanish & Finnish & Abundant & Medium & Rare & References \\
\hline Coriandrum sativum $\mathrm{L}$. & Coriander & Coriandre & Coriandolo & Koriander & $\begin{array}{l}\text { Cilantro, } \\
\text { culantro }\end{array}$ & Korianteri & & South-East Eu, & $\mathrm{D}$ & Ivanov, 1978; Crane et al., 1984 \\
\hline Crambe spp. & Sea-kale & & & Meerkohl & & & & & $\mathrm{NL}$ & \\
\hline Crataegus monogyna Jacq. & Hawthorn & $\begin{array}{l}\text { Aubépine à un } \\
\text { style }\end{array}$ & Biancospino & $\begin{array}{l}\text { Eingriffeliger } \\
\text { Weißdorn }\end{array}$ & Espino albar & $\begin{array}{l}\text { Tylppäliuskao- } \\
\text { rapihlaja }\end{array}$ & & E & $\mathrm{F}$ & $\begin{array}{l}\text { Maurizio and Schaper, 1994; Sáenz } \\
\text { Laín and Gómez Ferreras, 2000; } \\
\text { Clément, } 2002\end{array}$ \\
\hline Cynoglossum officinale $\mathrm{L}$. & Hound's-tongue & $\begin{array}{c}\text { Cynoglosse } \\
\text { officinal }\end{array}$ & $\begin{array}{l}\text { Lingua di } \\
\text { cane, } \\
\text { Vellutina }\end{array}$ & $\begin{array}{l}\text { Gebräuchliche } \\
\text { Hundszunge }\end{array}$ & Cinoglosa & Koirankieli & & & $\mathrm{NL}$ & Maurizio and Schaper, 1994 \\
\hline Daucus carota $\mathrm{L}$. & Carrot & Carotte & Carota & Möhre & Zanahoria & Porkkana & & & E, F, D, DK & $\begin{array}{l}\text { Maurizio and Louveaux, 1965; } \\
\text { Maurizio and Schaper, 1994; Sáenz } \\
\text { Laín and Gómez Ferreras, } 2000\end{array}$ \\
\hline $\begin{array}{l}\text { Diplotaxis erucoides } \\
\text { (Torner) DC. }\end{array}$ & $\begin{array}{l}\text { White wall } \\
\text { rocket }\end{array}$ & $\begin{array}{l}\text { Diplotaxis - } \\
\text { fausse } \\
\text { roquette }\end{array}$ & $\begin{array}{l}\text { Ruchetta (or } \\
\text { sanacciola) }\end{array}$ & $\begin{array}{l}\text { Rukenähnlicher } \\
\text { Doppelsame }\end{array}$ & r Jaramago & Hietasinapit & & E & I, F, P & $\begin{array}{l}\text { Maurizio and Louveaux, 1965; } \\
\text { Maurizio and Schaper, 1994; Persano } \\
\text { Oddo et al., 2000; Sáenz Laín and } \\
\text { Gómez Ferreras, 2000 }\end{array}$ \\
\hline $\begin{array}{l}\text { Dorycnium pentaphyllum } \\
\text { Scop. }\end{array}$ & & $\begin{array}{l}\text { Dorycnium à } \\
\text { cinq folioles }\end{array}$ & Trifoglino & $\begin{array}{l}\text { Fünfblättriger } \\
\text { Backenklee }\end{array}$ & $\begin{array}{l}\text { Mijediega, } \\
\text { boja caña- } \\
\text { mera }\end{array}$ & & & $\mathrm{E}$ & I & $\begin{array}{l}\text { Sáenz Laín and Gómez Ferreras, } \\
2000\end{array}$ \\
\hline $\begin{array}{l}\text { Echium brevirame } \\
\text { Sprague et Huch. }\end{array}$ & & & & $\begin{array}{l}\text { Kurzästiger } \\
\text { Natternkopf }\end{array}$ & Tajinaste & & & $\mathrm{E}^{*}$ & & $\begin{array}{l}\text { Sáenz Laín and Gómez Ferreras, } \\
2000\end{array}$ \\
\hline Echium spp. & $\begin{array}{l}\text { Purple viper's- } \\
\text { bugloss }\end{array}$ & Vipérine & Erba viperina & Natternkopf & $\begin{array}{l}\text { Vivorera, } \\
\text { chupamieles } \\
\text { sonaja }\end{array}$ & Neidonkieli & & $\mathrm{E}$ & I, NL, F & $\begin{array}{l}\text { Maurizio and Schaper, 1994; } \\
\text { Ricciardelli D'Albore, 1998; Bocquet, } \\
\text { 2001; Persano Oddo et al., 2000; Sáenz } \\
\text { Laín and Gómez Ferreras, } 2000\end{array}$ \\
\hline Echium virescens $\mathrm{DC}$ & & & & & Tajinaste & & & $\mathrm{E}^{*}$ & & \\
\hline Epilobium angustifolium $\mathrm{L}$. & $\begin{array}{c}\text { Rosebay } \\
\text { Willowherb }\end{array}$ & $\begin{array}{l}\text { Epilobe à } \\
\text { feuilles } \\
\text { étroites }\end{array}$ & $\begin{array}{l}\text { Epilobio, } \\
\text { garofanino } \\
\text { maggiore }\end{array}$ & $\begin{array}{l}\text { Wald-Weiden- } \\
\text { röschen }\end{array}$ & & Maitohorsma & & Fenno-Scand & & $\begin{array}{l}\text { Maurizio and Schaper, 1994; } \\
\text { Ricciardelli D'Albore, } 1998\end{array}$ \\
\hline Erica arborea $\mathrm{L}$. & Tree heath & $\begin{array}{l}\text { Bruyère } \\
\text { blanche ou } \\
\text { arborescente }\end{array}$ & Erica arborea & Baumheide & Brezo blanco & & $\mathrm{E}$ & I, F, HR & GR & $\begin{array}{l}\text { Louveaux, 1977; Gonnet and Vache, } \\
\text { 1998; Persano Oddo et al., 2000; } \\
\text { Sáenz Laín and Gómez Ferreras, } \\
\text { 2000; Clément, 2002; Ricciardelli } \\
\text { D'Albore, } 2003\end{array}$ \\
\hline Erica australis $\mathrm{L}$. & & & & & $\begin{array}{l}\text { Brezo rojo or } \\
\text { rubio }\end{array}$ & & $\mathrm{E}$ & & & $\begin{array}{l}\text { Sáenz Laín and Gómez Ferreras, } \\
2000\end{array}$ \\
\hline
\end{tabular}




\begin{tabular}{|c|c|c|c|c|c|c|c|c|c|c|}
\hline Latin name & English & French & Italian & German & Spanish & Finnish & Abundant & Medium & Rare & References \\
\hline Erica carnea $\mathrm{L}$. & Spring heather & $\begin{array}{l}\text { Bruyère } \\
\text { carnée }\end{array}$ & Erica carnea & Erika & & & & $\mathrm{F}$ & & Crane et al., 1984 \\
\hline Erica cinerea $\mathrm{L}$. & Bell heather & $\begin{array}{l}\text { Bruyère } \\
\text { cendrée }\end{array}$ & Erica cinerea & Grauheide & $\begin{array}{c}\text { Brezo } \\
\text { nazareno, } \\
\text { brezo gris, } \\
\text { ceniciento }\end{array}$ & $\begin{array}{l}\text { Harmaakello- } \\
\text { kanerva }\end{array}$ & $\mathrm{E}$ & F, BI & $P$ & $\begin{array}{l}\text { Louveaux, 1977; Gonnet and Vache, } \\
\text { 1998; Sáenz Laín and Gómez } \\
\text { Ferreras, 2000; Clément, } 2002\end{array}$ \\
\hline Erica manipuliflora Salisb. & & & & & & & & AL, GR, HR & & Ricciardelli D'Albore, 1985, 2003 \\
\hline Erica multiflora $\mathrm{L}$. & & & $\begin{array}{c}\text { Erica } \\
\text { multiflora }\end{array}$ & $\begin{array}{l}\text { Vielblütige } \\
\text { Heide }\end{array}$ & $\begin{array}{l}\text { Bruguera, } \\
\text { cipell }\end{array}$ & & $\mathrm{E}$ & HR & I & $\begin{array}{l}\text { Louveaux, 1977; Persano Oddo et al., } \\
\text { 2000; Sáenz Laín and Gómez } \\
\text { Ferreras, 2000; Ricciardelli } \\
\text { D'Albore, 2003 }\end{array}$ \\
\hline Erica scoparia $\mathrm{L}$. & & $\begin{array}{l}\text { Bruyère à } \\
\text { balai }\end{array}$ & Scopa & & $\begin{array}{l}\text { Brezo de } \\
\text { escobas }\end{array}$ & & E & & & $\begin{array}{l}\text { Sáenz Laín and Gómez Ferreras, } \\
2000\end{array}$ \\
\hline Erica umbellata $\mathrm{L}$. & & & & & $\begin{array}{l}\text { Quiruela, } \\
\text { mojariza }\end{array}$ & & $\mathrm{E}$ & & & $\begin{array}{l}\text { Sáenz Laín and Gómez Ferreras, } \\
2000\end{array}$ \\
\hline Erica vagans $\mathrm{L}$. & Cornish heath & $\begin{array}{c}\text { Bruyère } \\
\text { vagabonde }\end{array}$ & $\begin{array}{c}\text { Erica } \\
\text { vagabonda }\end{array}$ & Wanderheide & Rugón & & E & $\mathrm{F}$ & & $\begin{array}{l}\text { Louveaux, 1977; Sáenz Laín and } \\
\text { Gómez Ferreras, } 2000\end{array}$ \\
\hline Eryngium campestre $\mathrm{L}$. & Field eryngo & $\begin{array}{l}\text { Panicaut } \\
\text { champêtre }\end{array}$ & $\begin{array}{l}\text { Calcatrep- } \\
\text { pola }\end{array}$ & Feld-Mannstreu & $\begin{array}{l}\text { Cardo } \\
\text { corredor }\end{array}$ & & & $\mathrm{E}$ & $\mathrm{AL}$ & Ricciardelli D'Albore, 1985, 1998 \\
\hline $\begin{array}{l}\text { Eryobotrya japonica } \\
\text { (Thunb.) Lindley }\end{array}$ & $\begin{array}{l}\text { Loquat, Japa- } \\
\text { nese medlar }\end{array}$ & $\begin{array}{l}\text { Néflier du } \\
\text { Japon }\end{array}$ & $\begin{array}{l}\text { Nespolo del } \\
\text { Giappone }\end{array}$ & $\begin{array}{l}\text { Japanische } \\
\text { Wollmispel }\end{array}$ & Níspero & Japaninmispeli & & & $\mathrm{I}, \mathrm{E}$ & $\begin{array}{l}\text { Crane et al., 1984; Persano Oddo } \\
\text { et al., 2000; Sáenz Laín and Gómez } \\
\text { Ferreras, } 2000\end{array}$ \\
\hline Eucalyptus spp. & Gum tree & Eucalyptus & Eucalipto & Eukalyptus & Eucalipto & Eukalyptus & I, E, P & & GR, F & $\begin{array}{l}\text { Louveaux, 1970; Ricciardelli } \\
\text { D'Albore, 1998; Persano Oddo et al., } \\
\text { 2000; Sáenz Laín and Gómez Ferre- } \\
\text { ras, 2000; Piazza and Persano Oddo, } \\
2004\end{array}$ \\
\hline Euphorbia cyparissias $\mathrm{L}$. & Milk weed & $\begin{array}{l}\text { Euphorbe faux } \\
\text { cyprès }\end{array}$ & $\begin{array}{l}\text { Euforbia } \\
\text { cipressina }\end{array}$ & $\begin{array}{l}\text { Zypressenblät- } \\
\text { trige Wolfsmilch }\end{array}$ & & Tarhatyräkki & & & $\begin{array}{l}\text { South-East } \\
\text { Eu }\end{array}$ & Ricciardelli D’Albore, 1998 \\
\hline $\begin{array}{l}\text { Fagopyrum esculentum } \\
\text { Moench }\end{array}$ & Buckwheat & Sarrasin & $\begin{array}{l}\text { Grano } \\
\text { saraceno }\end{array}$ & $\begin{array}{c}\text { Echter } \\
\text { Buchweizen }\end{array}$ & $\begin{array}{c}\text { Trigo } \\
\text { sarraceno }\end{array}$ & Tattari & East Eu, PL & & $\begin{array}{l}\text { D, NL, A, F, } \\
\text { Fenno-Scand }\end{array}$ & $\begin{array}{l}\text { Crane et al., 1984; Bocquet, 1999b; } \\
\text { Clément, } 2002\end{array}$ \\
\hline $\begin{array}{l}\text { Galactites tomentosa } \\
\text { Moench }\end{array}$ & & & $\begin{array}{l}\text { Cardo, } \\
\text { scarlina }\end{array}$ & & $\begin{array}{l}\text { Cardota, } \\
\text { cardo } \\
\text { cuajaleches }\end{array}$ & & & I & $\mathrm{E}$ & Persano Oddo et al., 2000 \\
\hline
\end{tabular}




\begin{tabular}{|c|c|c|c|c|c|c|c|c|c|c|}
\hline Latin name & English & French & Italian & German & Spanish & Finnish & Abundant & Medium & Rare & References \\
\hline Glycine max (L.) Merrill & Soya & Soja & Soia & Sojabohne & Soja & Soijapapu & & & I & Crane et al., 1984 \\
\hline Gossypium hirsutum L. & Cotton & Coton & Cotone & Baumwolle & Algodonero & & & GR & $\begin{array}{l}\text { South-East } \\
\quad \text { Eu, E }\end{array}$ & $\begin{array}{l}\text { Crane et al., 1984; Ricciardelli } \\
\text { D’Albore, } 1998\end{array}$ \\
\hline Hedera helix $\mathrm{L}$. & Ivy & Lierre & Edera & Efeu & Hiedra & Muratti & & & I, E, F, HR & $\begin{array}{l}\text { Maurizio and Schaper, 1994; Gonnet } \\
\text { and Vache, 1998; Persano Oddo et al., } \\
\text { 2000; Clément, 2002; Ricciardelli } \\
\text { D'Albore, 2003 }\end{array}$ \\
\hline Hedysarum coronarium $\mathrm{L}$. & $\begin{array}{c}\text { French } \\
\text { honeysuckle }\end{array}$ & $\begin{array}{l}\text { Sainfoin à } \\
\text { bouquets }\end{array}$ & Sulla & Süßklee & $\begin{array}{l}\text { Hedísaro, } \\
\text { sulla }\end{array}$ & Punanätkin & & $\mathrm{I}, \mathrm{E}$ & $\mathrm{F}$ & $\begin{array}{l}\text { Crane et al., 1984; Ricciardelli } \\
\text { D'Albore, 1998; Persano Oddo et al., } \\
\text { 2000; Sáenz Laín and Gómez } \\
\text { Ferreras, } 2000\end{array}$ \\
\hline Helianthus annuиs $\mathrm{L}$. & Sunflower & Tournesol & Girasole & Sonnenblume & Girasol & $\begin{array}{l}\text { Auringon- } \\
\text { kukka }\end{array}$ & $\begin{array}{l}\text { I, E, F, East and } \\
\text { South-East Eu }\end{array}$ & D & $\begin{array}{l}\text { AL, GR, } \\
\text { Fenno-Scand }\end{array}$ & $\begin{array}{l}\text { Ricciardelli D’Albore, 1985, 1998; } \\
\text { Persano Oddo et al., 2000; Sáenz } \\
\text { Laín and Gómez Ferreras, 2000; } \\
\text { Clément, 2002; Piazza and Persano } \\
\text { Oddo, } 2004\end{array}$ \\
\hline $\begin{array}{l}\text { Hirschfeldia incana (L.) } \\
\text { Lagr.- F. }\end{array}$ & Hoary mustard & $\begin{array}{l}\text { Hirschfeldie } \\
\text { grisâtre }\end{array}$ & $\begin{array}{l}\text { Senape } \\
\text { cornuta }\end{array}$ & Graukohl & Relinchón & & & $\mathrm{E}^{*}$ & & \\
\hline Hyресоuт sp. & & & Cornacchina & & Zadorija & & & E & & $\begin{array}{l}\text { Sáenz Laín and Gómez Ferreras, } \\
2000\end{array}$ \\
\hline Inula viscosa $(\mathrm{L}$.$) Aiton$ & $\begin{array}{l}\text { Woody } \\
\text { fleabane }\end{array}$ & $\begin{array}{l}\text { Inule } \\
\text { visqueuse }\end{array}$ & Enula & Alant & Inula & & & & $\mathrm{I}, \mathrm{E}$ & $\begin{array}{l}\text { Ricciardelli D’Albore, } 1998, \\
\text { Persano Oddo et al., } 2000\end{array}$ \\
\hline $\begin{array}{l}\text { Lavandula angustifolia } \\
\text { Miller }\end{array}$ & Lavender & Lavande vraie ${ }^{\mathrm{L}}$ & $\begin{array}{l}\text { Lavanda vera } \\
\text { (o spigo) }\end{array}$ & Echter Lavendel & Lavanda & Laventeli & $\mathrm{E}$ & $\mathrm{F}$ & & $\begin{array}{l}\text { Maurizio and Louveaux, 1965; } \\
\text { Maurizio and Schaper, 1994; Persano } \\
\text { Oddo et al., } 2000\end{array}$ \\
\hline $\begin{array}{l}\text { L. } \mathrm{x} \text { intermedia Emeric ex } \\
\text { Loiselieur }\end{array}$ & $\begin{array}{l}\text { English } \\
\text { lavender }\end{array}$ & Lavandin & $\begin{array}{l}\text { Lavanda } \\
\text { ibrida }\end{array}$ & Lavandin & Lavandín & & $\mathrm{F}$ & $\mathrm{E}, \mathrm{HR}$ & $\begin{array}{l}\text { South-East } \\
\text { Eu }\end{array}$ & $\begin{array}{l}\text { Maurizio and Louveaux, 1965; } \\
\text { Louveaux, 1970; Maurizio and } \\
\text { Schaper, 1994; Ricciardelli } \\
\text { '’Albore, 1999, 2003; Persano Oddo } \\
\text { et al., 2000; Clément, 2002; Piazza } \\
\text { and Persano Oddo, 2004 }\end{array}$ \\
\hline $\begin{array}{l}\text { Lavandula latifolia } \\
\text { Medikus }\end{array}$ & Spike lavender & $\begin{array}{l}\text { Lavande à } \\
\text { larges feuilles }\end{array}$ & Spigo & Großer Speik & Espliego & & $\mathrm{E}$ & & & $\begin{array}{l}\text { Maurizio and Louveaux, 1965; } \\
\text { Louveaux, 1970; Maurizio and } \\
\text { Schaper, 1994; Sáenz Laín and } \\
\text { Gómez Ferreras, } 2000\end{array}$ \\
\hline Lavandula stoechas $\mathrm{L}$. & $\begin{array}{l}\text { French } \\
\text { lavender }\end{array}$ & $\begin{array}{l}\text { Lavande } \\
\text { stechas }\end{array}$ & $\begin{array}{l}\text { Lavanda } \\
\text { selvatica }\end{array}$ & Schopf-lavendel & Cantueso & & E, P & & $\mathrm{I}, \mathrm{F}$ & $\begin{array}{l}\text { Persano Oddo et al., 2000; Sáenz } \\
\text { Laín and Gómez Ferreras, 2000; } \\
\text { Clément, } 2002\end{array}$ \\
\hline
\end{tabular}




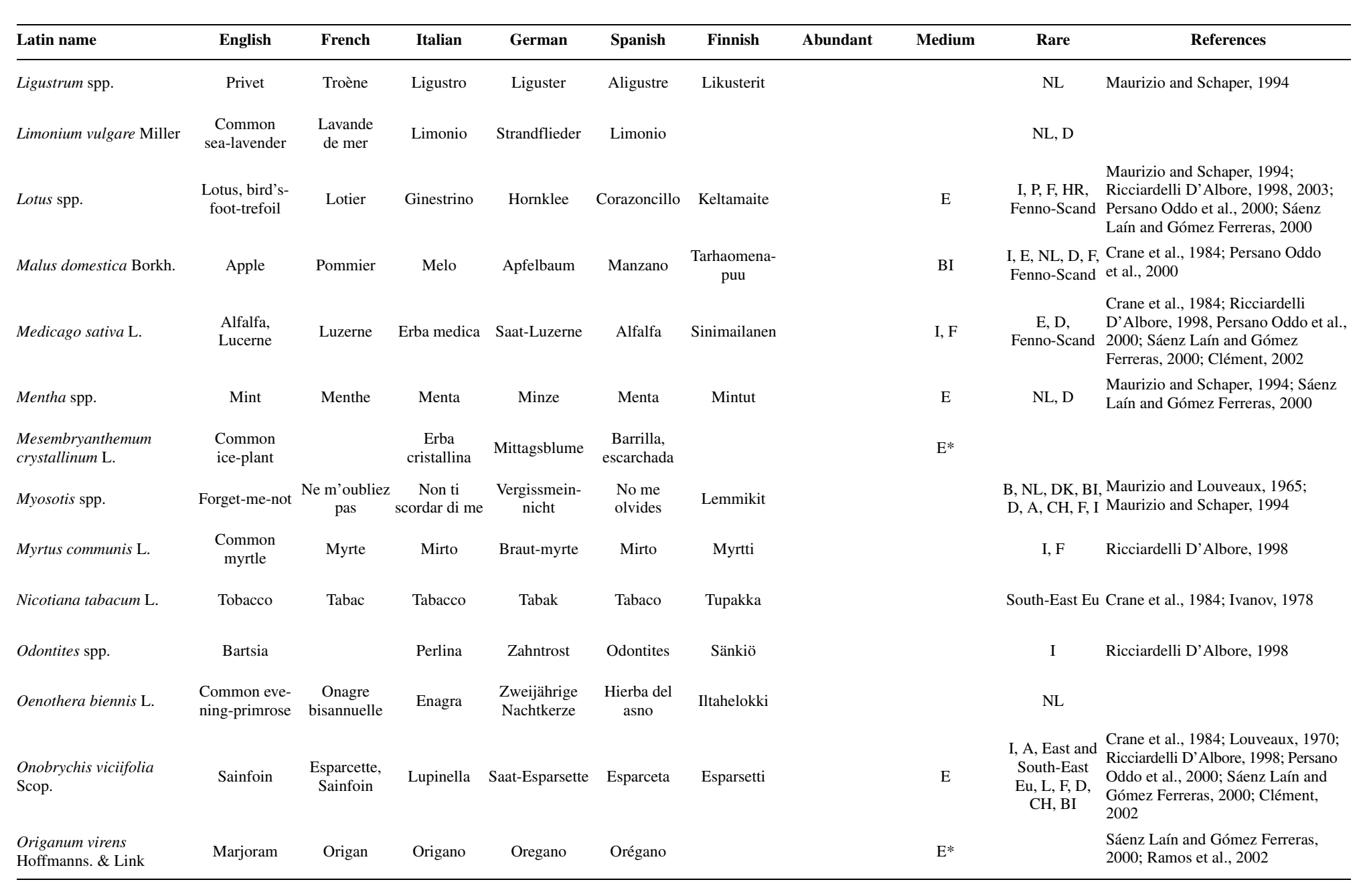




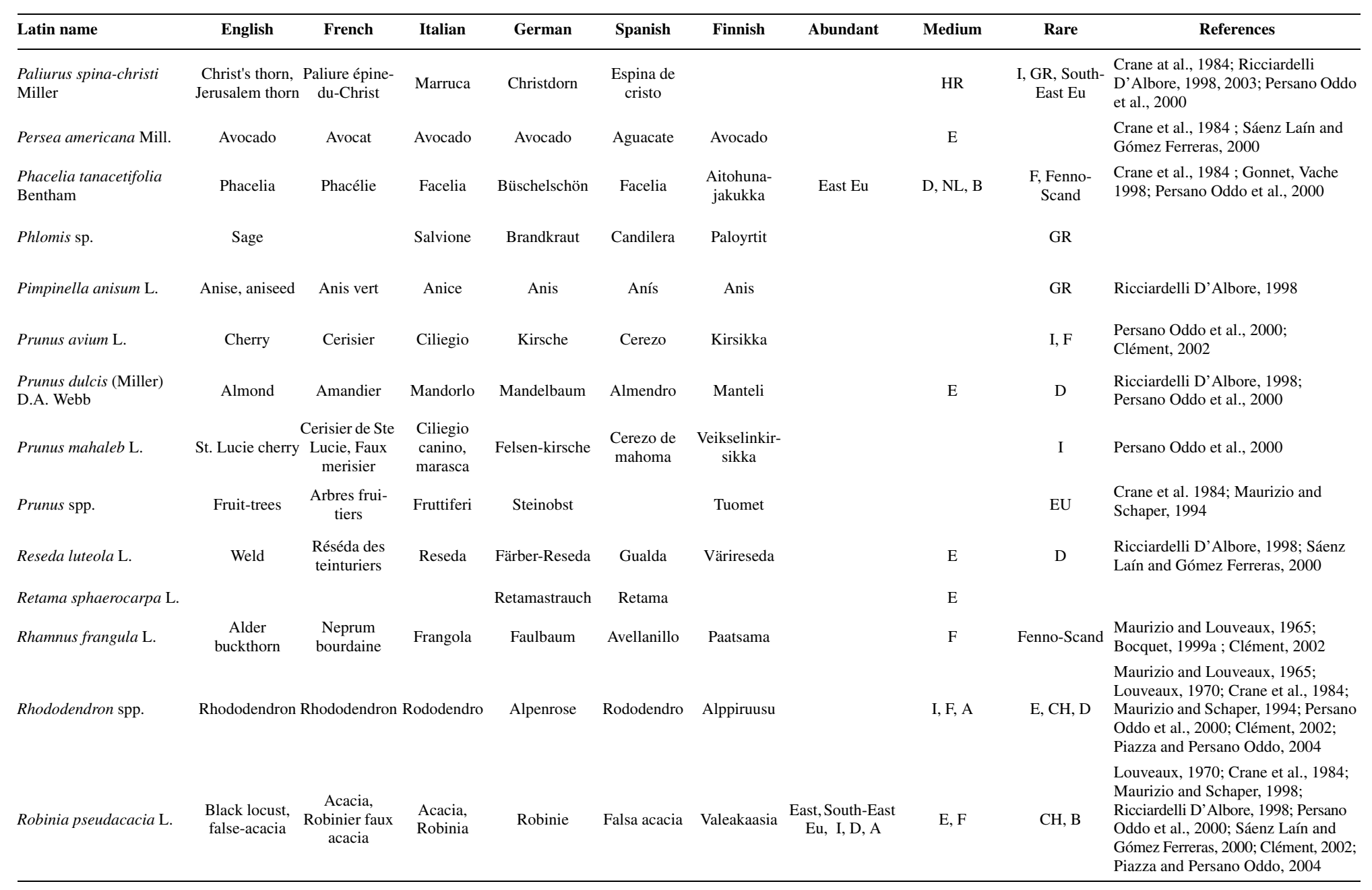




\begin{tabular}{|c|c|c|c|c|c|c|c|c|c|c|}
\hline Latin name & English & French & Italian & German & Spanish & Finnish & Abundant & Medium & Rare & References \\
\hline Rosmarinus officinalis $\mathrm{L}$. & Rosemary & Romarin & Rosmarino & Rosmarin & Romero & Rosmariini & E & $\mathrm{I}, \mathrm{F}, \mathrm{HR}$ & & $\begin{array}{l}\text { Louveaux, 1970; Crane at al., 1984; } \\
\text { Maurizio and Schaper, 1994; } \\
\text { Ricciardelli D'Albore, 1998, 2003; } \\
\text { Persano Oddo et al., 2000; Sáenz } \\
\text { Laín and Gómez Ferreras, 2000; } \\
\text { Clément, 2002; Piazza and Persano } \\
\text { Oddo, 2004 }\end{array}$ \\
\hline Rubus spp. & Blackberry & Ronces & Rovo & Himbeere & $\begin{array}{c}\text { Zarza, } \\
\text { zarzamora }\end{array}$ & Vatukka & Fenno-Scand & E, P & $\begin{array}{l}\text { AL, I, NL, D, } \\
\text { CH, BI, F }\end{array}$ & $\begin{array}{l}\text { Crane et al., 1984; Ricciardelli } \\
\text { D'Albore, 1985, 1998; Maurizio and } \\
\text { Schaper, 1994; Persano Oddo et al., } \\
\text { 2000; Sáenz Laín and Gómez } \\
\text { Ferreras, 2000; Clément, } 2002\end{array}$ \\
\hline Salix spp. & Willow & Saules & Salice & Weide & Sauce & Paju & & $\begin{array}{l}\text { E, HR, } \\
\text { Fenno-Scand }\end{array}$ & $\mathrm{NL}, \mathrm{CH}, \mathrm{D}, \mathrm{F}$ & $\begin{array}{l}\text { Crane et al., 1984; Maurizio and } \\
\text { Schaper, 1994; Persano Oddo et al., } \\
\text { 2000; Clément, 2002; Ricciardelli } \\
\text { D'Albore, } 2003\end{array}$ \\
\hline Salvia officinalis $\mathrm{L}$. & Sage & Sauge & Salvia & Salbei & Salvia & Ryytisalvia & & HR & GR & $\begin{array}{l}\text { Crane et al., 1984; Maurizio and } \\
\text { Schaper, 1994; Ricciardelli } \\
\text { D'Albore, 1998, } 2003\end{array}$ \\
\hline Satureja montana $\mathrm{L}$. & Winter savory & $\begin{array}{l}\text { Sariette des } \\
\text { montagnes }\end{array}$ & $\begin{array}{c}\text { Santoreggia } \\
\text { montana }\end{array}$ & $\begin{array}{c}\text { Winter- } \\
\text { Bohnenkraut }\end{array}$ & Ajedrea & Talvikynteli & & E & $\mathrm{I}, \mathrm{F}, \mathrm{HR}$ & $\begin{array}{l}\text { Crane et al., 1984; Maurizio and } \\
\text { Schaper, 1994; Ricciardelli } \\
\text { D'Albore, 1998, 2003; Persano Oddo } \\
\text { et al., } 2000\end{array}$ \\
\hline Sideritis montana $\mathrm{L}$. & & $\begin{array}{l}\text { Crapaudine } \\
\text { des montagnes }\end{array}$ & $\begin{array}{l}\text { Stregonia } \\
\text { montana }\end{array}$ & Berg-Gliedkraut & & & & & $\mathrm{F}$ & \\
\hline Sideritis syriaca $\mathrm{L}$. & & & Stregonia & & Sideritide & & & & I, E, GR & Persano Oddo et al., 2000 \\
\hline Sinapis arvensis $\mathrm{L}$. & Charlock & $\begin{array}{l}\text { Moutarde des } \\
\text { champs }\end{array}$ & Senape & Acker - Senf & & Rikkasinppi & & & & $\begin{array}{l}\text { Maurizio and Louveaux, 1965; } \\
\text { Maurizio and Schaper, } 1994\end{array}$ \\
\hline Solidago virgaurea $\mathrm{L}$. & Golden rod & $\begin{array}{l}\text { Solidage, } \\
\text { verge d'or }\end{array}$ & Verga d'oro & $\begin{array}{l}\text { Gewöhnliche } \\
\text { Goldrute }\end{array}$ & Vara de oro & Kultapiisku & & & I, E, F & $\begin{array}{l}\text { Maurizio and Schaper, 1994; } \\
\text { Ricciardelli D'Albore, 1998, } \\
\text { Persano Oddo et al., } 2000\end{array}$ \\
\hline Sorbus aucuparia $\mathrm{L}$. & Rowan & $\begin{array}{c}\text { Sorbier des } \\
\text { oiseleurs }\end{array}$ & $\begin{array}{l}\text { Sorbo degli } \\
\text { uccellatori }\end{array}$ & Eberesche & $\begin{array}{l}\text { Serval de los } \\
\text { cazadores }\end{array}$ & Kotipihlaja & & & Fenno-Scand & \\
\hline $\begin{array}{l}\text { Spartocytisus supranu- } \\
\text { bius [L.] Webb \& Berth }\end{array}$ & & & & & $\begin{array}{l}\text { Retama del } \\
\text { teide }\end{array}$ & & & $\mathrm{E}^{*}$ & & \\
\hline Stachys аппиа $\mathrm{L}$. & $\begin{array}{l}\text { Annual yellow- } \\
\text { woundwort }\end{array}$ & $\begin{array}{c}\text { Epiaire } \\
\text { annuelle }\end{array}$ & $\begin{array}{l}\text { Erba uggia, } \\
\text { erba strega }\end{array}$ & $\begin{array}{l}\text { Einjühriger } \\
\text { Ziest }\end{array}$ & Estachis & Keltapähkämö & & & $\mathrm{I}, \mathrm{E}$ & $\begin{array}{l}\text { Maurizio and Schaper, 1994; } \\
\text { Persano Oddo et al., } 2000\end{array}$ \\
\hline
\end{tabular}




\begin{tabular}{|c|c|c|c|c|c|c|c|c|c|c|}
\hline Latin name & English & French & Italian & German & Spanish & Finnish & Abundant & Medium & Rare & References \\
\hline Taraxacum officinale group & Dandelion & Pissenlit & Tarassaco & Löwenzahn & $\begin{array}{l}\text { Diente de } \\
\text { león }\end{array}$ & Voikukka & & $\begin{array}{l}\text { I, CH, B, D, F, } \\
\text { A, Fenno-Scand }\end{array}$ & E & $\begin{array}{l}\text { Maurizio and Louveaux, 1965; } \\
\text { Maurizio and Schaper, 1994; Persano } \\
\text { Oddo et al., 2000; Sáenz Laín and } \\
\text { Gómez Ferreras, 2000; Clément, } \\
\text { 2002; Piazza and Persano Oddo, } \\
\text { 2004 }\end{array}$ \\
\hline Teucrium marum $\mathrm{L}$. & Germander & & Maro & Katzenkraut & Teucrio & & & & I & Persano Oddo et al., 2000 \\
\hline Teucrium spp. & Germander & Germandrée & Maro & Gamander & & & & & D & $\begin{array}{l}\text { Maurizio and Louveaux, 1965; } \\
\text { Maurizio and Schaper, } 1994\end{array}$ \\
\hline $\begin{array}{l}\text { Thymus capitatus (L.) } \\
\text { Hofmgg. et Lk. }\end{array}$ & Thyme & Thym & $\begin{array}{c}\text { Timo } \\
\text { arbustivo }\end{array}$ & Thymian & $\begin{array}{l}\text { Tomillo } \\
\text { cabezudo }\end{array}$ & & GR & $\mathrm{AL}, \mathrm{I}, \mathrm{HR}$ & E & $\begin{array}{l}\text { Maurizio and Louveaux, 1965; } \\
\text { Louveaux, 1970; Maurizio and } \\
\text { Schaper, 1994; Ricciardelli } \\
\text { D'Albore, 1985, 1998, 2003; } \\
\text { Persano Oddo et al., 2000; Piazza and } \\
\text { Persano Oddo, 2004 }\end{array}$ \\
\hline $\begin{array}{l}\text { Thymus serpyllum } \\
\text { (sensu lato) }\end{array}$ & $\begin{array}{l}\text { Breckland } \\
\text { thyme }\end{array}$ & $\begin{array}{l}\text { Thym } \\
\text { serpolet }\end{array}$ & Timo serpillo & & $\begin{array}{l}\text { Tomillo } \\
\text { serpol }\end{array}$ & $\begin{array}{l}\text { Kangasaju- } \\
\text { ruoho }\end{array}$ & & & E, I, F & $\begin{array}{l}\text { Maurizio and Louveaux, 1965; } \\
\text { Maurizio and Schaper, 1994; } \\
\text { Ricciardelli D'Albore, 1998; Persano } \\
\text { Oddo et al., 2000; Clément, } 2002\end{array}$ \\
\hline Thymus vulgaris $\mathrm{L}$. & Garden thyme & Thym vulgaire & e $\begin{array}{c}\text { Timo } \\
\text { maggiore }\end{array}$ & Thymian & $\begin{array}{l}\text { Tomillo } \\
\text { vulgar }\end{array}$ & Timjami & E & & $\mathrm{F}$ & $\begin{array}{l}\text { Sáenz Laín and Gómez Ferreras, } \\
\text { 2000; Clément, } 2002\end{array}$ \\
\hline Tilia spp. & Lime & Tilleul & Tiglio & Linde & Tilo & Lehmus & $\begin{array}{c}\text { D, NL, PL, } \\
\text { East, South-East } \\
\text { Eu }\end{array}$ & $\mathrm{I}, \mathrm{E}, \mathrm{F}$ & $\begin{array}{l}\text { CH, B, } \\
\text { Fenno-Scand }\end{array}$ & $\begin{array}{l}\text { Louveaux, 1970; Maurizio and } \\
\text { Louveaux, 1965; Maurizio and } \\
\text { Schaper, 1994; Ricciardelli } \\
\text { D'Albore, 1998; Persano Oddo et al., } \\
\text { 2000; Sáenz Laín and Gómez Ferre- } \\
\text { ras, 2000; Clément, 2002; Piazza and } \\
\text { Persano Oddo, } 2004\end{array}$ \\
\hline Trifolium alexandrinum $\mathrm{L}$. & Egyptian clover & $\begin{array}{c}\text { Trèfle } \\
\text { d'Alexandrie }\end{array}$ & $\begin{array}{c}\text { Trifoglio } \\
\text { alessandrino }\end{array}$ & $\begin{array}{l}\text { Alexandriner- } \\
\text { Klee }\end{array}$ & Trébol & Egyptinapila & & & I & Persano Oddo et al., 2000 \\
\hline Trifolium incarnatum $\mathrm{L}$. & Crimson clover & $\begin{array}{c}\text { Trèfle } \\
\text { incarnat }\end{array}$ & $\begin{array}{l}\text { Trifoglio } \\
\text { incarnato }\end{array}$ & Inkarnat-klee & $\begin{array}{l}\text { Trébol } \\
\text { encarnado }\end{array}$ & Veriapila & & & I, F, East Eu & $\begin{array}{l}\text { Maurizio and Louveaux, 1965; } \\
\text { Maurizio and Schaper, 1994; } \\
\text { Ricciardelli D'Albore, 1998; } \\
\text { Persano Oddo et al., } 2000\end{array}$ \\
\hline Trifolium pratense $\mathrm{L}$. & Red clover & Trèfle des prés & is $\begin{array}{c}\text { Trifoglio } \\
\text { violetto }\end{array}$ & Rotklee & $\begin{array}{l}\text { Trébol de los } \\
\text { prados }\end{array}$ & Puna-apila & & E & $\begin{array}{l}\text { I, DK, D, GR, } \\
\text { F, Fenno-Scand }\end{array}$ & $\begin{array}{l}\text { Maurizio and Louveaux, 1965; } \\
\text { Maurizio and Schaper, 1994; } \\
\text { Ricciardelli D'Albore, 1998 }\end{array}$ \\
\hline
\end{tabular}




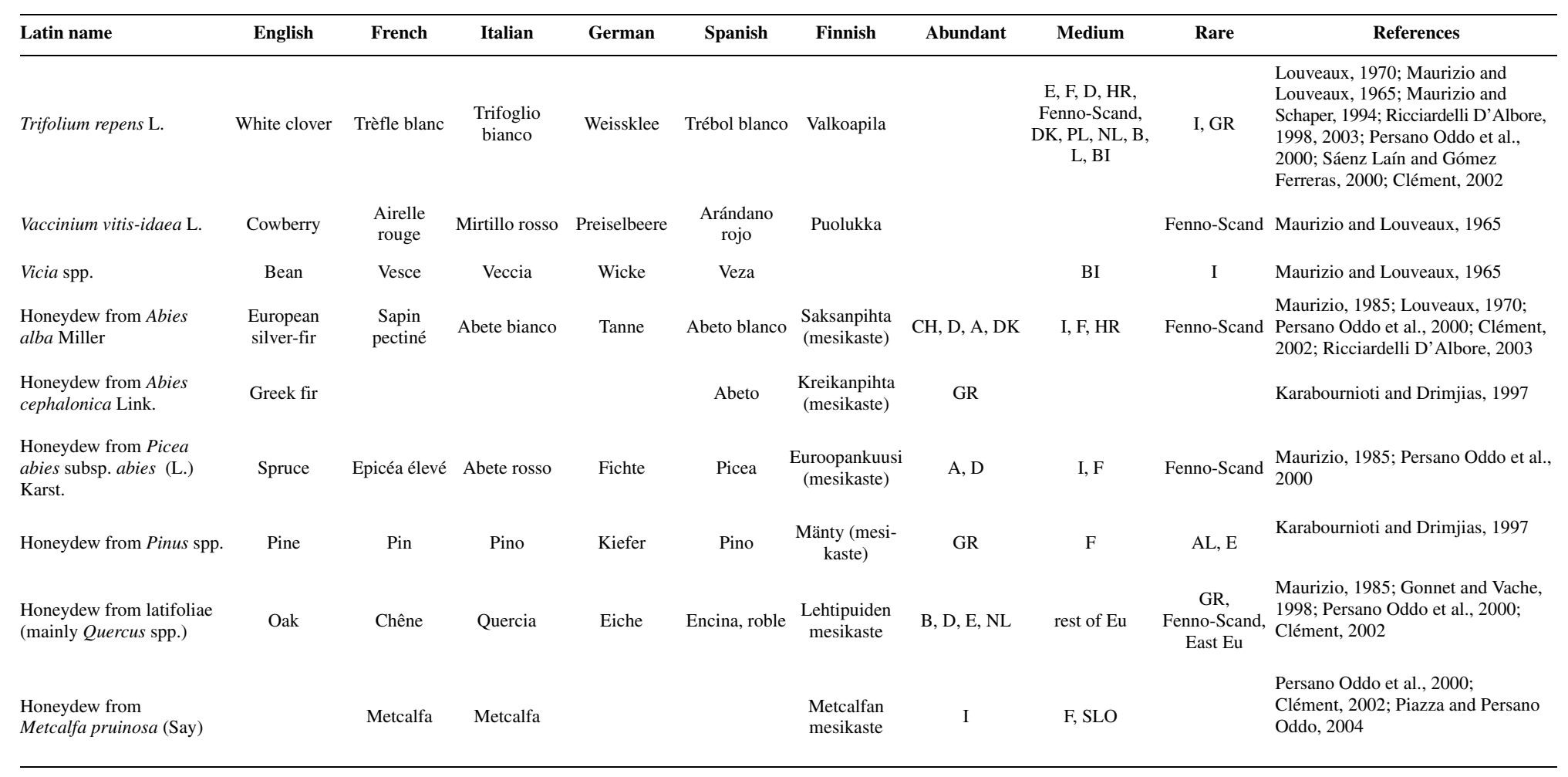




\section{REFERENCES}

Bocquet M. (1999a) Le miel de bourdaine. Nature et composition, principales caractéristiques organoleptiques, Bull. Tech. Apic. 26, 91-92.

Bocquet M. (1999b) Le miel de sarrasin. Nature et composition. Principales caractéristiques organoleptiques, Bull. Tech. Apic. 26, 143-144.

Bocquet M. (2001) Le miel de vipérine : nature et composition, principales caractéristiques organoleptiques, Bull. Tech. Apic. 28, 96-97.

Clément H. (ed.) (2002) Le traité Rustica de l'apiculture, Rustica Ed., Paris.

Crane E., Walker P. (1985) Important honeydew sources and their honeys, Bee World 66, 105-112.

Crane E., Walker P., Day R. (1984) Directory of important world honey sources. Nectar plants, International Bee Research Association, London.

Gonnet M., Vache G. (1998) Analyse sensorielle descriptive de quelques miels monofloraux de France et d'Europe, Syndicat National d'Apiculture, Paris.

Ivanov T. (1978) Composition and properties of Bulgarian honey. Dissertation, Ministry of Food and Agriculture, Bee Research Station, Sofia, Bulgaria (in Bulgarian).

Karabournioti S., Drimjias N. (1997) Some physicochemical characteristics of Greek monofloral honeys, Apiacta 32, 44-50

Kloft W., Kunkel H. (1985) Waldtracht und Waldhonig in der Imkerei, Ehrenwirth Verlag, München.

Liebig G. (1999) Die Waldtracht. Entstehung - Beobachtung - Prognose, G. Liebig, Stuttgart.

Louveaux J. (1970) Annexes microphotographiques aux méthodes officielles d'analyse. Tome III, Atlas photographique d'analyse pollinique des miels, Service de la répression des fraudes et du contrôle de la qualité, Paris.

Louveaux J. (1977) Les bruyères et leur miel, Bull. Tech. Apic. 4, 31-36.
Maurizio A. (1965) Honigtau-Honigtauhonig, Waldhonigbuch Ehrenwirth, München.

Maurizio A. (1985) Honigtau-Honigtauhonig, in: Kloft W., Kunkel H. (Eds.), Waldtracht und Waldhonig in der Imkerei, Ehrenwirth, München, pp. 268-295.

Maurizio A., Louveaux J. (1965) Pollens de plantes mellifères d'Europe, Union des Groupements Apicoles Français, Paris.

Maurizio A., Schaper F. (1994) Das Trachtpflanzenbuch. Nektar und Pollen - die wichtigsten Nahrungsquellen der Honigbiene, Ehrenwirth, München.

Persano Oddo L., Piro R. (2004) Main European unifloral honeys: descriptive sheets, Apidologie 35 (Suppl. 1), S38-S81.

Persano Oddo L., Sabatini A.G., Accorti M., Colombo R., Marcazzan G.L., Piana M.L., Piazza M.G., Pulcini P. (2000) I mieli uniflorali italiani. Nuove schede di caratterizzazione, Istituto Sperimentale per la Zoologia Agraria, Ministero delle Politiche Agricole e Forestali, Roma.

Piazza M.G., Persano Oddo L. (2004) Bibliographical review of the main European unifloral honeys, Apidologie 35 (Suppl. 1), S94-S111.

Prota R., Floris I., Papoff C.M. (1997) Comparazione delle caratteristiche chimico-fisiche di mieli della Sardegna e della Corsica, Apic. Mod. 88, 51-59.

Quayle L. (1997) Honeybee forage - borage: its cultivation, uses and value to bees, Beekeep. Q., 22-24.

Ramos I.L.S., Perez B.M., Ferreras C.G. (2002) Pollen spectra of different unifloral honeys from $\mathrm{La}$ Palma (Canary Islands, Spain), Grana 41, 48-57.

Ricciardelli D’Albore G. (1985) Lo spettro pollinico dei mieli di Albania, Apicoltura 1, 1-33.

Ricciardelli D'Albore G. (1998) Mediterranean melissopalynology, Institute of Agricultural Entomology, University of Perugia.

Ricciardelli D'Albore G. (2003) Conosciamo meglio i mieli della Croazia, Apitalia XXX, 13-14.

Sáenz Laín C., Gómez Ferreras C. (2000) Mieles Españolas, Ed. Mundi-Prensa. 